\title{
Editorial
}

\section{Serum levels of hyaluronic acid and chondroitin sulfate as a non-invasive method to evaluate healing after cartilage repair procedures}

\author{
Andreas H Gomoll \\ Department of Orthopedic Surgery, Brigham and Women's Hospital, Harvard Medical School, 75 Francis Street, Boston, MA 02115, USA
}

Corresponding author: Andreas H Gomoll, agomoll@yahoo.com

Published: 3 July 2009

Arthritis Research \& Therapy 2009, 11:118 (doi:10.1186/ar2730)

This article is online at http://arthritis-research.com/content/11/4/118

(c) 2009 BioMed Central Ltd

See related research by Nganvongpanit et al., http://arthritis-research.com/content/11/3/R78

\begin{abstract}
Magnetic resonance imaging remains the only non-invasive method to assess the quality of cartilage repair procedures, but ideally would be complemented by other modalities, particularly blood tests. Nganvongpanit and colleagues investigated serum levels of hyaluronic acid (HA) and chondroitin sulfate (CS) for their correlation with tissue quality after cartilage repair with autologous chondrocytes versus subchondral drilling in a dog model. They reported better tissue quality in animals treated with chondrocyte implantation. Serum levels correlated with the histological score of biopsy samples: CS showed a negative $(r=-0.69)$ and HA a positive $(r=+0.46)$ correlation. Many questions remain to be answered before serum markers can provide a reliable, noninvasive tool to assess tissue quality, but these data provide an important foundation for additional research.
\end{abstract}

In the previous issue of Arthritis Research \& Therapy, Nganvongpanit and colleagues [1], of Chiang Mai University in Thailand, investigated the potential use of serum biomarkers, such as hyaluronic acid (HA) and chondroitin sulfate (CS), to evaluate healing after cartilage repair procedures. They randomly assigned dogs to treatment with autologous chondrocyte implantation $(\mathrm{ACl})$ versus subchondral drilling (SD) and followed the animals for 24 weeks post-operatively with multiple blood draws and a cartilage biopsy at final follow-up.

Cartilage defects are a common diagnosis, encountered in over $60 \%$ of knee arthroscopies [2]. While the natural history and pathophysiology of cartilage defects remain controversial, a significant number of patients present with symptoms that warrant surgical intervention. These patients undergo various cartilage repair procedures to repair the damaged articular surfaces, including microfracture, osteochondral autografting, and $\mathrm{ACl}$. Progress in the field of cartilage repair has been impeded in part by the relative lack of adequate instruments to evaluate the quality of the reparative tissue. While histological evaluation is desirable, researchers have found it difficult to recruit patients for a second surgical procedure to harvest a tissue biopsy solely for research purposes. Imaging techniques, especially magnetic resonance imaging (MRI), have made significant progress in recent years. Certain cartilage-specific techniques such as delayed gadolinium-enhanced MRI of cartilage (dGEMRIC) and T1-rho and T2-mapping have promise to assess tissue quality by indirectly measuring glycosaminoglycan content $[3,4]$. However, these techniques are associated with substantial cost and potential risk to the patient from contrast exposure; therefore, the development of alternative noninvasive techniques is desirable. In particular, blood tests, which could be repeated multiple times with minimal discomfort to the patient, would present an ideal method to investigate the maturation of repair tissue after cartilage repair. Beyond the scientific benefit of comparing the relative time courses of healing after different repair techniques, once thresholds are established, biomarkers could provide clinical guidance regarding the point when patients might return to full activities.

In their article, Nganvongpanit and colleagues investigated the use of monoclonal antibodies and enzyme-linked immunosorbent assay to quantify serum levels of CS and HA, respectively, in a dog model. They followed two groups treated with either SD or autologous chondrocytes (ACs) for 24 weeks, with blood draws at baseline and every 6 weeks

$\mathrm{AC}=$ autologous chondrocyte $\mathrm{ACl}=$ autologous chondrocyte implantation; $\mathrm{CS}=$ chondroitin sulfate $\mathrm{HA}=$ hyaluronic acid; $\mathrm{MRI}=$ magnetic resonance imaging; $S D=$ subchondral drilling. 
thereafter. Other endpoints included the gross visual evaluation of the reparative tissue as well as histologic grading. Animals treated with ACs demonstrated better visual and histological appearance than those treated with drilling. Three of the five $\mathrm{AC}$ biopsies were near normal, and the other two showed at least $50 \%$ fill and peripheral integration of the repair site. In the SD group, three of five samples demonstrated complete degeneration, and the other two only inconsistent fill and no peripheral integration with the surrounding articular surface. Histologically, both groups demonstrated some fibrocartilage; however, the AC group also showed hyaline cartilage compared with fibrous tissue in the SD group.

Interestingly, serum levels of CS and HA demonstrated different trends at final follow-up after 24 weeks: CS had a strong negative correlation with histological scores $(r=-0.69)$, while HA was positively correlated $(r=+0.46)$. In the AC group, CS levels trended downward over time, a finding the authors interpret as a reflection of the normalizing proteoglycan turnover due to a successful repair with maturing tissue. In the SD group, however, levels remained high, possibly reflecting the progressive damage of the surrounding cartilage seen in these samples. Overall, HA levels also decreased from baseline, with relatively higher values in samples with better histological scores, potentially a sign of normalization of joint homeostasis.

This study provided two important findings. First, it added to the mounting evidence of improved histological outcomes with cell-based therapy, such as $\mathrm{ACl}$ [5], over marrowstimulation techniques, such as SD or microfracture. Second, the authors describe two potential candidate factors to follow tissue maturation and healing: HA and CS. Many questions remain to be addressed, such as the correlation of marker levels with defect size, number, and location as well as possible differences between chondral and osteochondral defects and patient gender, age, or weight. However, these preliminary results are promising and provide a foundation for future research.

In conclusion, while these findings require larger, confirmatory studies (ideally in human patients), they hold promise for non-invasive monitoring after cartilage repair procedures. Reliable, reproducible, and relatively inexpensive methods to evaluate the quality and maturation of reparative tissue will substantially advance the field of cartilage repair. These tests would potentially enable investigators and industry to develop new technologies aimed at repairing articular cartilage, assist surgeons to select the appropriate procedure for any given patient, and post-operatively, allow an individualized determination of when it is safe for the patient to return to higher levels of activity.

\section{Competing interests}

The author declares that they have no competing interests.

\section{References}

1. Nganvongpanit $K$, Pothacharoen $P$, Chaochird $P$, Klunklin $K$, Warrit K, Settakorn J, Pattamapaspong N, Luevitoonvechkij S, Arpornchayanon O, Kongtawelert P, Pruksakorn D: Prospective evaluation of serum biomarker levels and cartilage repair by autologous chondrocyte transplantation and subchondral drilling in a canine model. Arthritis Res Ther 2009, 11:R78.

2. Curl WW, Krome J, Gordon ES, Rushing J, Smith BP, Poehling GG: Cartilage injuries: a review of 31,516 knee arthroscopies. Arthroscopy 1997, 13:456-460.

3. Recht MP, Goodwin DW, Winalski CS, White LM: MRI of articular cartilage: revisiting current status and future directions. AJR Am J Roentgenol 2005, 185:899-914.

4. Potter HG, Foo LF: Magnetic resonance imaging of articular cartilage: trauma, degeneration, and repair. Am J Sports Med 2006, 34:661-677.

5. Saris DB, Vanlauwe J, Victor J, Haspl M, Bohnsack M, Fortems Y Vandekerckhove B, Almqvist KF, Claes T, Handelberg F, Lagae K, van der Bauwhede J, Vandenneucker $H$, Yang KG, Jelic M, Verdonk R, Veulemans N, Bellemans J, Luyten FP: Characterized chondrocyte implantation results in better structural repair when treating symptomatic cartilage defects of the knee in a randomized controlled trial versus microfracture. Am J Sports Med 2008, 36:235-246. 